\title{
Rare Lepton-Number-Violating W Decays at the LHC: CP Violation
}

\section{David London}

Physique des Particules, Université de Montréal, 1375 Avenue Thérèse-Lavoie-Roux, Montréal, QC, Canada H2V 0B3

\section{Abstract}

Some models of leptogenesis involve a nearly-degenerate pair of heavy Majorana neutrinos $N_{1,2}$ whose masses can be small, $O(\mathrm{GeV})$. There can be heavy-light neutrino mixing parametrized by $\left|B_{\ell N}\right|^{2}=10^{-5}$, which leads to the rare lepton-number-violating decay $W^{ \pm} \rightarrow \ell_{1}^{ \pm} \ell_{2}^{ \pm}\left(q^{\prime} \bar{q}\right)^{\mp}$. With contributions to this decay from both $N_{1}$ and $N_{2}$, a CP-violating rate difference between the decay and its CP-conjugate can be generated. In this talk, I describe the prospects for measuring such a CP asymmetry $A_{\mathrm{CP}}$ at the LHC. I consider three versions of the LHC - HL-LHC, HE-LHC, FCC-hh - and show that, for $5 \mathrm{GeV} \leq M_{N} \leq$ $80 \mathrm{GeV}$, small values of the $\mathrm{CP}$ asymmetry can be measured at $3 \sigma$, in the range $1 \% \lesssim A_{\mathrm{CP}} \lesssim 15 \%$.

Keywords: lepton-number violation, $W$ decays at the LHC, CP violation, leptogenesis models, light sterile neutrinos DOI: $10.31526 /$ ACP.BSM-2021.15

Talk based on work done in collaboration with Fatemeh Najafi and Jacky Kumar, Ref. [1].

\section{INTRODUCTION}

One of the fundamental mysteries in particle physics - indeed, in all of physics - is the origin of the baryon asymmetry of the universe (BAU). The only thing we know for sure about the BAU is that its generation requires the three Sakharov conditions:

(i) baryon-number violation, (ii) CP violation, (iii) processes that take place out of equilibrium [2]. One popular explanation is leptogenesis. Here, a lepton-number asymmetry is created through CP-violating, lepton-number-violating processes. This is then converted to a baryon-number asymmetry via sphalerons processes [3, 4], which conserve $B-L$.

Another mystery is neutrino masses, which are known to be nonzero, but very small. What is the origin of these neutrino masses? And are neutrinos Dirac or Majorana particles? If they are Majorana, low-energy lepton-number-violating processes such as neutrinoless double-beta decay may be observable.

A common scenario in leptogenesis models, which also touches the question of neutrino masses, is the appearance of a pair of nearly-degenerate heavy sterile neutrinos $N_{1}$ and $N_{2}$. Leptogenesis can then be produceed through CP-violating decays of the heavy neutrinos [5,6], or via neutrino oscillations [7, 8]. We will see both of these effects below.

In the seesaw mechanism $[9,10,11]$ with one left-handed ( $\mathrm{LH})$ and one right-handed (RH, sterile) neutrino, the mass matrix takes the form

$$
M=\left(\begin{array}{cc}
0 & m_{D} \\
m_{D} & m_{R}
\end{array}\right),
$$

leading to

$$
m_{v}=\frac{m_{D}^{2}}{m_{R}}, \quad m_{N}=m_{R}
$$

The standard choice for the entries in the mass matrix is $m_{D} \sim m_{t}, m_{R} \sim 10^{15} \mathrm{GeV}$. But there are other possibilities, e.g., $m_{D} \sim m_{e}$, $m_{R} \sim 1 \mathrm{TeV}$.

With three LH and three RH neutrinos, there are more free parameters in the mass matrix (three $m_{D} \mathrm{~s}$ and three $m_{R} \mathrm{~s}$ ). A complete scan of the parameter space reveals that it is possible to obtain three ultralight neutrinos $v_{i}$ and three heavy Majorana neutrinos $N_{i}$, with $N_{1}$ and $N_{2}$ nearly degenerate and with masses of $O(\mathrm{GeV})$ [12].

The flavour and mass eigenstates are related via

$$
v_{\ell}=\sum_{j=1}^{3} B_{\ell j} v_{j}+\sum_{i=1}^{3} B_{\ell N_{i}} N_{i}
$$

Here the $B_{\ell N_{i}}$ parametrize the heavy-light neutrino mixing. The point is the following. With $B_{\ell N_{i}} \neq 0$, there are $W-\ell-N_{i}$ couplings. And if $M_{N}<M_{W}$, one can have the decay $W^{-} \rightarrow \ell_{1}^{-} N_{i}$, with (i) $N_{i} \rightarrow \ell_{2}^{-} \ell_{3}^{+} v_{\ell_{3}}, \ell_{2}^{-}\left(q^{\prime} \bar{q}\right)^{+}$or (ii) $N_{i} \rightarrow \ell_{2}^{+} \ell_{3}^{-} \bar{v}_{\ell_{3}}$. Decays of type (i) are lepton-number violating $(\mathrm{LNV}, \Delta L=2$ ), while decays of type (ii) are lepton-number conserving (LNC, $\Delta L=0)$. Searches for such decays constrain the mixing parameters to be

$$
\left|B_{\ell N}\right|^{2} \leq 10^{-5} \quad(\ell=e, \mu)
$$


for $1 \mathrm{GeV} \leq m_{N} \leq 80 \mathrm{GeV}[13]$.

The idea that there can be a pair of nearly-degenerate Majorana neutrinos with masses of $O(\mathrm{GeV})$ has led a number of authors to examine the prospects for observing CP-violating LNV processes in the decays of mesons $[14,15,16,17,18,19,20,21]$ and $\tau$ leptons [22, 23]. For example, the decay $B^{ \pm} \rightarrow D^{0} \ell_{1}^{ \pm} \ell_{2}^{ \pm} \pi^{\mp}$ is considered in Ref. [19]. It occurs via $B^{ \pm} \rightarrow D^{0} W^{* \pm}\left(\rightarrow \ell_{1}^{ \pm} N_{i}\right)$, with $N_{i} \rightarrow \ell_{2}^{ \pm} W^{* \mp}\left(\rightarrow \pi^{\mp}\right)$.

The key point here is that we can search for similar effects in the decays of real Ws at the LHC, in $W^{-} \rightarrow \ell_{1}^{-} \ell_{2}^{-}\left(f^{\prime} \bar{f}\right)^{+}$. This decay has already been studied extensively as a signal of LNV. Here we push further and examine the prospects for measuring $\mathrm{CP}$ violation in this decay.

As noted above, in $W^{-} \rightarrow \ell_{1}^{-} N_{i}$, if the $N_{i}$ decays leptonically, the final state can be $\ell_{1}^{-} \ell_{2}^{-} \ell_{3}^{+} v_{\ell_{3}}$ (LNV) or $\ell_{1}^{-} \ell_{2}^{+} \ell_{3}^{-} \bar{v}_{\ell_{3}}$ (LNC). Since the final-state (anti)neutrino is not detected, these are indistinguishable. However, we want to focus on pure LNV decays, so in our study we consider only $W^{-} \rightarrow \ell_{1}^{-} \ell_{2}^{-}\left(q^{\prime} \bar{q}\right)^{+}$. A difference between the rates of $W^{-} \rightarrow \ell_{1}^{-} \ell_{2}^{-}\left(q^{\prime} \bar{q}\right)^{+}$and its CP-conjugate decay $W^{+} \rightarrow \ell_{1}^{+} \ell_{2}^{+}\left(q^{\prime} \bar{q}\right)^{-}$is a signal of CP violation.

\section{CP VIOLATION - REVIEW}

Suppose that the decay $W^{-} \rightarrow F$, where $F$ is the final state, has two contributing amplitudes, $A$ and $B$ :

$$
A_{\text {tot }}=A+B=|A| e^{i \phi_{A}} e^{i \delta_{A}}+|B| e^{i \phi_{B}} e^{i \delta_{B}},
$$

where $\phi_{A, B}$ and $\delta_{A, B}$ are $\mathrm{CP}$-odd and $\mathrm{CP}$-even phases, respectively. The $\mathrm{CP}$ asymmetry is

$$
\begin{aligned}
A_{\mathrm{CP}} & =\frac{B R\left(W^{-} \rightarrow F\right)-B R\left(W^{+} \rightarrow \bar{F}\right)}{B R\left(W^{-} \rightarrow F\right)+B R\left(W^{+} \rightarrow \bar{F}\right)} \\
& =\frac{2|A||B| \sin \left(\phi_{A}-\phi_{B}\right) \sin \left(\delta_{A}-\delta_{B}\right)}{|A|^{2}+|B|^{2}+2|A||B| \cos \left(\phi_{A}-\phi_{B}\right) \cos \left(\delta_{A}-\delta_{B}\right)} .
\end{aligned}
$$

From this we see that a nonzero $A_{\mathrm{CP}}$ requires the two contributing amplitudes to have different $\mathrm{CP}$-odd phases $\left(\phi_{A}-\phi_{B} \neq 0\right)$ and different CP-even phases $\left(\delta_{A}-\delta_{B} \neq 0\right)$. In addition, $A_{\mathrm{CP}}$ is sizeable only when the two amplitudes are of similar size $(|A| \sim|B|)$.

In $W^{-} \rightarrow \ell_{1}^{-} \ell_{2}^{-}\left(q^{\prime} \bar{q}\right)^{+}$, the two amplitudes are $W^{-} \rightarrow \ell_{1}^{-} \bar{N}_{1,2}$, with each of $\bar{N}_{1,2}$ decaying to $\ell_{2}^{-}\left(q^{\prime} \bar{q}\right)^{+}$. Here $\phi_{1}=$ $\arg \left[B_{\ell_{1} N_{1}} B_{\ell_{2} N_{1}}\right]$ and $\phi_{2}=\arg \left[B_{\ell_{1} N_{2}} B_{\ell_{2} N_{2}}\right]$, so that $\phi_{1}-\phi_{2}$ can be nonzero.

There are two sources of $\mathrm{CP}$-even phases. First, the $N_{i}$ propagator is proportional to

$$
\begin{aligned}
\frac{1}{\left(p_{N}^{2}-M_{N_{i}}^{2}\right)+i M_{N_{i}} \Gamma_{N_{i}}} & =\frac{1}{\sqrt{\left(p_{N}^{2}-M_{N_{i}}^{2}\right)^{2}+M_{N_{i}}^{2} \Gamma_{N_{i}}^{2}}} e^{i \eta_{i}} \\
\text { with } \tan \eta_{i} & =\frac{-M_{N_{i}} \Gamma_{N_{i}}}{\left(p_{N}^{2}-M_{N_{i}}^{2}\right)} .
\end{aligned}
$$

As $N_{1}$ and $N_{2}$ do not have exactly the same mass, this leads to $\eta_{1}-\eta_{2} \neq 0$. For example, if $\eta_{1}=-\pi / 2$ (i.e., $N_{1}$ is on-shell), then $\left|\eta_{2}\right|<\pi / 2$. This is resonant CP violation.

Note also that, since the $N_{i}$ are nearly degenerate, the two amplitudes are of similar size, so that $A_{\mathrm{CP}}$ can be sizeable.

Second, there can be oscillations of heavy neutrinos. The time evolution of a heavy $N_{i}$ mass eigenstate involves the factor $e^{-i E_{i} t}$, where $E_{i}$ is the energy of the $N_{i}$ in the rest frame of the decaying $W$. Once again, since $M_{N_{1}} \neq M_{N_{2}}$, we have $E_{1} \neq E_{2}$, which gives different $e^{-i E_{i} t}$ factors. This is another source of a CP-even phase difference, and can also lead to CP violation. 


\section{3. $\mathcal{M}\left(W^{-} \rightarrow \ell_{1}^{-} \bar{N}_{I}, N_{I} \rightarrow \ell_{2}^{-} W^{*+}\left(\rightarrow\left(Q^{\prime} \bar{Q}\right)^{+}\right)\right.$}

The Feynman diagram for $W^{-} \rightarrow \ell_{1}^{-} \ell_{2}^{-}\left(q^{\prime} \bar{q}\right)^{+}$via an intermediate $N_{i}$ is shown in Fig. 1.

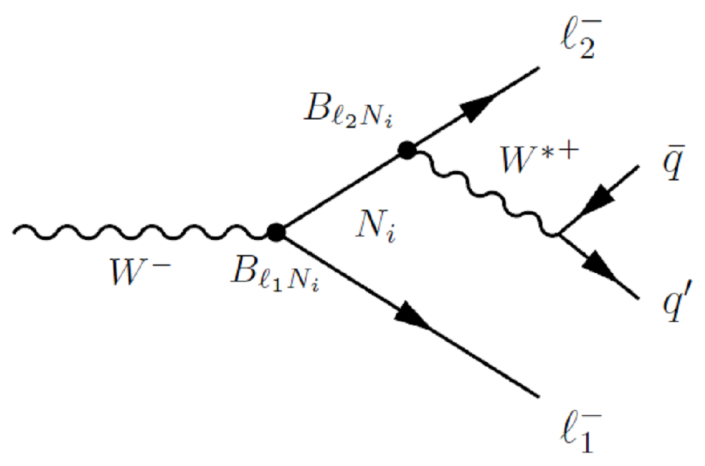

FIGURE 1: Diagram for $W^{-} \rightarrow \ell_{1}^{-} \ell_{2}^{-}\left(q^{\prime} \bar{q}\right)^{+}$via an intermediate $N_{i}$. There is no arrow on the $N_{i}$ line because it is a Majorana particle and the decay is fermion-number violating.

Because this decay receives contributions from $N_{i}=N_{1}$ and $N_{2}$, and since the two neutrinos cannot be on shell simultaneously, we must include the heavy neutrino propagator in the amplitude. In addition, although the neutrino is produced as $\bar{N}_{i}$, it actually decays as $N_{i}$, leading to the fermion-number-violating and LNV process $W^{-} \rightarrow \ell_{1}^{-} \ell_{2}^{-}\left(q^{\prime} \bar{q}\right)^{+}$. This implies that (i) conjugate fields will be involved in the amplitudes, and (ii) the amplitudes will be proportional to the neutrino mass.

The full amplitudes are $\mathcal{M}_{i}^{--} \equiv \mathcal{M}\left(W^{-} \rightarrow \ell_{1}^{-} \bar{N}_{i}, \bar{N}_{i} \rightarrow N_{i}, N_{i} \rightarrow \ell_{2}^{-} W^{*+}\left(\rightarrow\left(q^{\prime} \bar{q}\right)^{+}\right)\right.$. Writing $\mathcal{M}_{i}^{--}=\mathcal{M}_{i}^{\mu v} \epsilon_{\mu} j_{v}$, where $\epsilon_{\mu}$ is the polarization of the initial $W^{-}$and $j_{v}=\frac{g}{\sqrt{2}} \bar{q} \gamma_{v} P_{L} q^{\prime}$ is the current of final-state particles to which $W^{*+}$ decays, we have

$$
\begin{aligned}
\mathcal{M}_{i}^{\mu \nu} & =\bar{\ell}_{1} \gamma^{\mu} P_{L}\left(\frac{g}{\sqrt{2}} B_{\ell_{1} N_{i}}\right) N_{i} \times e^{-\Gamma_{i} t / 2} e^{-i E_{i} t} \times \bar{\ell}_{2} \gamma^{v} P_{L}\left(\frac{g}{\sqrt{2}} B_{\ell_{2} N_{i}}\right) N_{i} \\
& \rightarrow \frac{\frac{g^{2}}{2} B_{\ell_{1} N_{i}} B_{\ell_{2} N_{i}} M_{i} e^{-\Gamma_{i} t / 2} e^{-i E_{i} t}}{p_{N}^{2}-M_{i}^{2}+i \Gamma_{i} M_{i}} L^{\mu \nu},
\end{aligned}
$$

where $L^{\mu v}=\bar{\ell}_{1} \gamma^{\mu} \gamma^{v} P_{R} \ell_{2}^{c}$. In the first line, the first term is the amplitude for $W^{-} \rightarrow \ell_{1}^{-} \bar{N}_{i}$, the second term is the time dependence of the $N_{i}$ state, and the third term is the amplitude for $N_{i} \rightarrow \ell_{2}^{-} W^{*+}$. The $e^{-i E_{i} t}$ factor is due to the quantum-mechanical evolution of the $N_{i}$ state (neutrino oscillations). The CP-odd phase is found in $B_{\ell_{1} N_{i}} B_{\ell_{2} N_{i}}$, while the CP-even phase arises from the $e^{-i E_{i} t}$ and $i \Gamma_{i} M_{i}$ factors.

The total amplitude is $\mathcal{M}^{\mu \nu}=\mathcal{M}_{1}^{\mu \nu}+\mathcal{M}_{2}^{\mu \nu}$. Writing $B_{\ell_{1} N_{1}} B_{\ell_{2} N_{1}} \equiv B_{1} e^{i \phi_{1}}$ and $B_{\ell_{1} N_{2}} B_{\ell_{2} N_{2}} \equiv B_{2} e^{i \phi_{2}}$, we have

$$
\mathcal{M}^{\mu \nu}=\frac{g^{2}}{2}\left(\frac{M_{1} B_{1} e^{i \phi_{1}} e^{-\Gamma_{1} t / 2} e^{-i E_{1} t}}{p_{N}^{2}-M_{1}^{2}+i \Gamma_{1} M_{1}}+\frac{M_{2} B_{2} e^{i \phi_{2}} e^{-\Gamma_{2} t / 2} e^{-i E_{2} t}}{p_{N}^{2}-M_{2}^{2}+i \Gamma_{2} M_{2}}\right) L^{\mu \nu}
$$

Note that the two contributing amplitudes have different CP-odd phases ( $\phi_{1}$ and $\left.\phi_{2}\right)$ and (two sources of) different CP-even phases $\left(i \Gamma_{1} M_{1}\right.$ vs. $i \Gamma_{2} M_{1}$ and $e^{-i E_{1} t}$ vs. $\left.e^{-i E_{2} t}\right)$. We therefore expect to find a CP asymmetry.

Using this expression, we (i) compute $\left|\mathcal{M}^{\mu v}\right|^{2}$ using the narrow-width approximation, (ii) integrate over time (our goal is not the measurement of the neutrino oscillations), (iii) perform the phase-space integrals, and (iv) construct $A_{\mathrm{CP}}$. 


\section{4. $A_{\mathrm{CP}}$}

With the simplifying assumption that $B_{1}=B_{2}$, we find

$$
A_{\mathrm{CP}}=\frac{2(2 y-x) \sin \delta \phi}{\left(1+x^{2}\right)\left(1+4 y^{2}\right)+2(1-2 x y) \cos \delta \phi},
$$

where

$$
x \equiv \frac{\Delta E}{\Gamma}, \quad y \equiv \frac{\Delta M}{\Gamma}, \quad \text { with } \quad x=y \frac{M_{N}}{M_{W}} .
$$

Comparing Eqs. (6) and (10), we see that $x$ and $y$ each play the role of the CP-even phase-difference term $\sin \left(\delta_{A}-\delta_{B}\right) \cdot x$ arises from neutrino oscillations (hence the factor $\Delta E$ ), while $y$ is due to the neutrino propagator $(\Delta M)$.

We note that $y$ is always present; $x$ is generally subdominant, except for large values of $M_{N}$. Given that $|2 y-x| \leq|2 y|$, this implies that that, as $|x|$ increases, $A_{\mathrm{CP}}$ decreases. We therefore expect to see smaller CP-violating effects for larger values of $M_{N}$. $M_{N}$.

In order to estimate the potential size of $A_{\mathrm{CP}}$, we set $\delta \phi=\pi / 2$. In Fig. 2, we plot $A_{\mathrm{CP}}$ as a function of $y$, for various values of

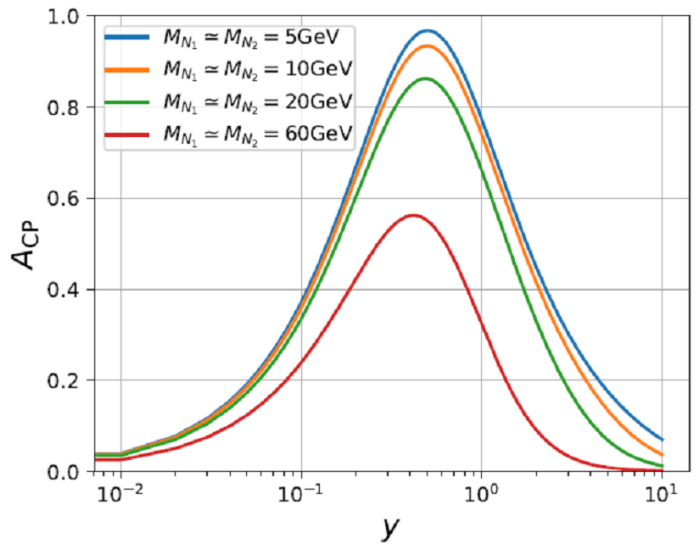

FIGURE 2: Value of $A_{\mathrm{CP}}$ as a function of $y$, for $\delta \phi=\pi / 2$ and for various values of $M_{N}$. For negative values of $y, A_{\mathrm{CP}} \rightarrow-A_{\mathrm{CP}}$.

From this plot, we note the following features:

- Large values of $\left|A_{\mathrm{CP}}\right|(\geq 0.9)$ can be produced for light $M_{N}$.

- Maximal values of $\left|A_{\mathrm{CP}}\right|$ are found when $y \simeq \pm \frac{1}{2}$, with $\left|A_{\mathrm{CP}}\right|$ decreasing for larger/smaller values of $|y|$.

- As expected, the size of $\left|A_{\mathrm{CP}}\right|$ decreases as $M_{N}$ increases, with $\left|A_{\mathrm{CP}}\right|_{\max }<0.6$ for larger values of $M_{N}$. (Even so, these values of $\left|A_{\mathrm{CP}}\right|$ are not that small.)

Note in passing: the observation that $\mathrm{CP}$ violation is maximal when $y \simeq \pm \frac{1}{2}$ allows us to quantify how degenerate the "nearlydegenerate heavy sterile neutrinos" must be. Using $y \equiv \Delta M / \Gamma$, we find that, for $M_{N}=10 \mathrm{GeV}, \Delta M=O\left(10^{-14}\right) \mathrm{GeV}$.

\section{EXPERIMENTAL PROSPECTS}

In order to measure $A_{\mathrm{CP}}$, one has to compare $N_{--}$(the number of events of $\left.W^{-} \rightarrow \ell_{1}^{-} \ell_{2}^{-}\left(q^{\prime} \bar{q}\right)^{+}\right)$and $N_{++}$(the number of events of $\left.W^{+} \rightarrow \ell_{1}^{+} \ell_{2}^{+}\left(q \bar{q}^{\prime}\right)^{-}\right)$. However, one must also take into account the fact that, because $p p$ collisions are involved at the LHC, and because protons do not contain an equal number of up- and down-type quarks and antiquarks, the number of $W^{-}$and $W^{+}$bosons produced is not equal. This can be done by measuring

$$
A_{\mathrm{CP}}=\frac{R_{W} N_{--}^{p p}-N_{++}^{p p}}{R_{W} N_{--}^{p p}+N_{++}^{p p}},
$$

where $N_{--}^{p p}$ and $N_{++}^{p p}$ are the number of observed events of $p p \rightarrow X W^{-}\left(\rightarrow \ell_{1}^{-} \ell_{2}^{-}\left(q^{\prime} \bar{q}\right)^{+}\right)$and $p p \rightarrow X W^{+}\left(\rightarrow \ell_{1}^{+} \ell_{2}^{+}\left(\bar{q}^{\prime} q\right)^{-}\right)$, respectively, and

$$
R_{W}=\frac{\sigma\left(p p \rightarrow W^{+} X\right)}{\sigma\left(p p \rightarrow W^{-} X\right)}
$$

measured to be $R_{W}=1.295 \pm 0.003$ (stat) \pm 0.010 (syst) at $\sqrt{s}=13 \mathrm{TeV}$ [24]. Presumably, $R_{W}$ can be measured with equally good precision (if not better) at higher energies. 
Now, given an $A_{\mathrm{CP}}$, the number of events required to show it is nonzero at $n \sigma$ is

$$
N_{\text {events }}=\frac{n^{2}}{A_{C P}^{2} \epsilon},
$$

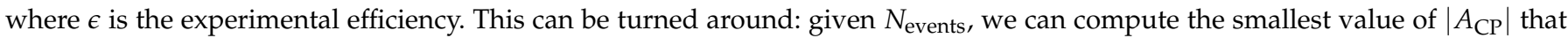
can be measurable at $n \sigma$.

In our study, we consider three versions of the LHC: (i) the high-luminosity LHC (HL-LHC, $\sqrt{s}=14$ TeV), (ii) the high-energy LHC (HE-LHC, $\sqrt{s}=27 \mathrm{TeV})$, and (iii) the future circular collider (FCC-hh, $\sqrt{s}=100 \mathrm{TeV})$. We implement the model in FeynRules $[25,26]$ and use MadGraph [27] to generate events. We take $\left|B_{\ell N}\right|^{2} \leq 10^{-5}$.

Note that $N_{\text {events }}$ is not whole story. What we really want is the number of measurable events. To be specific, we require that the sterile neutrinos actually decay in the detector. With this in mind, it is necessary to look at the $N$ lifetime and determine what percentage of the heavy neutrinos actually decay in the detector. This was done by the CMS Collaboration in its search for $W^{-} \rightarrow \ell_{1}^{-} \ell_{2}^{-}\left(f^{\prime} \bar{f}\right)^{+}$[28]. They found that, for $M_{N}=1 \mathrm{GeV}, 5 \mathrm{GeV}$ and $10 \mathrm{GeV}$, the multiplicative reduction factor was $10^{-3}, 0.1$ and $\simeq 1$, respectively.

In its searches for heavy Majorana neutrinos at the $\sqrt{s}=8 \mathrm{TeV}$ LHC using the final state $\ell_{1}^{-} \ell_{2}^{-} j j$ [29, 30], the CMS Collaboration found that their overall efficiency was $\sim 1 \%$. Using this efficiency in our estimates, we obtain the results given in Table 1.

\begin{tabular}{|c|c|c|c|}
\hline \multicolumn{4}{|c|}{ Minimum $A_{\mathrm{CP}}$ measurable at $3 \sigma$} \\
\hline Machine & $M_{N}=5 \mathrm{GeV}$ & $M_{N}=10 \mathrm{GeV}$ & $M_{N}=50 \mathrm{GeV}$ \\
\hline HL-LHC & $15.0 \%$ & $4.8 \%$ & $7.4 \%$ \\
HE-LHC & $5.1 \%$ & $1.6 \%$ & $2.5 \%$ \\
FCC-hh & $2.1 \%$ & $0.7 \%$ & $1.0 \%$ \\
\hline
\end{tabular}

TABLE 1: Minimum value of $A_{\mathrm{CP}}$ measurable at $3 \sigma$ at the HL$\operatorname{LHC}(\sqrt{s}=14 \mathrm{TeV})$, HE-LHC $(\sqrt{s}=27 \mathrm{TeV})$ and FCC-hh $(\sqrt{s}=100 \mathrm{TeV})$. Results are given for $M_{N}=5 \mathrm{GeV}$ (reduction factor $=0.1), M_{N}=10 \mathrm{GeV}$ (no reduction factor), and $M_{N}=50 \mathrm{GeV}$ (no reduction factor).

We note that

- As LHC increases in energy and integrated luminosity, smaller values of $A_{\mathrm{CP}}$ are measurable.

- At a given machine, the measurable $A_{\mathrm{CP}}$ decreases as $M_{N}$ increases. (But there is a reduction factor due to the $N$ lifetime for small $M_{N}$.)

- The most promising results are for $M_{N}=10 \mathrm{GeV}$, but in all cases reasonably small values of $A_{\mathrm{CP}}$ can be probed.

\section{SUMMARY}

In many leptogenesis models, a lepton-number asymmetry arises through CP-violating decays of a pair of nearly-degenerate heavy neutrinos $N_{1}$ and $N_{2}$. What is particularly intriguing is that the masses of $N_{1,2}$ can be small, $O(\mathrm{GeV})$.

In general, there can be a (small) heavy-light neutrino mixing. This leads to LNV processes at the $\mathrm{LHC}_{\mathrm{Luch}}$ as $W^{ \pm} \rightarrow$ $\ell_{1}^{ \pm} \ell_{2}^{ \pm}\left(q^{\prime} \bar{q}\right)^{\mp}$. A CP-violating rate asymmetry $A_{\mathrm{CP}}$ between the $W^{-}$and $W^{+}$decays can arise due to the interference of the $N_{1}$ and $N_{2}$ contributions. The different $W-\ell-N_{1}$ and $W-\ell-N_{2}$ couplings produce the CP-odd phase difference; The CP-even phase difference is generated via propagator effects or oscillations of the heavy neutrinos.

If such an LNV decay were observed, this would of course be very exciting. But the next step would be to try to understand the underlying origin of the decay. One important piece of information would be to look at CP violation in the decay, and this is what we have studied.

We consider $5 \mathrm{GeV} \leq M_{N} \leq 80 \mathrm{GeV}$ and examine three versions of the LHC: (i) HL-LHC $(\sqrt{s}=14 \mathrm{TeV})$, (ii) HE-LHC $(\sqrt{s}=27$ $\mathrm{TeV})$, (iii) FCC-hh $(\sqrt{s}=100 \mathrm{TeV})$. The most promising result is for the FCC-hh with $M_{N}=10 \mathrm{GeV}$. Here $A_{\mathrm{CP}}=\mathrm{O}(1 \%)$ is measurable. But even in the worst case, the HL-LHC with $M_{N}=5 \mathrm{GeV}$, an $A_{\mathrm{CP}}=O(10 \%)$ can be measured.

\section{ACKNOWLEDGEMENTS}

This work was financially supported by NSERC of Canada. 


\section{References}

[1] F. Najafi, J. Kumar and D. London, JHEP 04, 021 (2021) doi:10.1007/JHEP04(2021)021 [arXiv:2011.03686 [hep-ph]].

[2] A. D. Sakharov, "Violation of CP Invariance, C asymmetry, and baryon asymmetry of the universe," Sov. Phys. Usp. 34, no.5, 392-393 (1991) doi:10.1070/PU1991v034n05ABEH002497

[3] G. 't Hooft, "Symmetry Breaking Through Bell-Jackiw Anomalies," Phys. Ref. Lett. 37, 8-11 (1976) doi:10.1103/PhysRevLett.37.8

[4] G. 't Hooft, "Computation of the Quantum Effects Due to a Four-Dimensional Pseudoparticle," Phys. Ref. D 14, 3432-3450 (1976) [erratum: Phys. Ref. D 18, 2199 (1978)] doi:10.1103/PhysRevD.14.3432

[5] A. Pilaftsis, "CP violation and baryogenesis due to heavy Majorana neutrinos," Phys. Ref. D 56, 5431-5451 (1997) doi:10.1103/PhysRevD.56.5431 [arXiv:hep-ph/9707235 [hep-ph]].

[6] A. Pilaftsis and T. E. J. Underwood, “Resonant leptogenesis," Nucl. Phys. B 692, 303-345 (2004) doi:10.1016/j.nuclphysb.2004.05.029 [arXiv:hepph/0309342 [hep-ph]].

[7] E. K. Akhmedov, V. A. Rubakov and A. Y. Smirnov, "Baryogenesis via neutrino oscillations," Phys. Ref. Lett. 81, 1359-1362 (1998) doi:10.1103/PhysRevLett.81.1359 [arXiv:hep-ph/9803255 [hep-ph]].

[8] L. Canetti, M. Drewes, T. Frossard and M. Shaposhnikov, "Dark Matter, Baryogenesis and Neutrino Oscillations from Right Handed Neutrinos," Phys. Ref. D 87, 093006 (2013) doi:10.1103/PhysRevD.87.093006 [arXiv:1208.4607 [hep-ph]].

[9] M. Gell-Mann, P. Ramond and R. Slansky, "Complex Spinors and Unified Theories," Conf. Proc. C 790927, 315-321 (1979) [arXiv:1306.4669 [hep-th]].

[10] T. Yanagida in Proceedings: Workshop on the Unified Theories and the Baryon Number in the Universe, O. Sawada and A. Sugamoto, eds. Natl. Lab. High Energy Phys., Tsukuba, Japan, 1979.

[11] R. N. Mohapatra and G. Senjanovic, "Neutrino Mass and Spontaneous Parity Nonconservation," Phys. Ref. Lett. 44, 912 (1980) doi:10.1103/PhysRevLett.44.912

[12] For example, see L. Canetti, M. Drewes and B. Garbrecht, "Probing leptogenesis with GeV-scale sterile neutrinos at LHCb and Belle II," Phys. Ref. D 90, no.12, 125005 (2014) doi:10.1103/PhysRevD.90.125005 [arXiv:1404.7114 [hep-ph]]

[13] F. F. Deppisch, P. S. Bhupal Dev and A. Pilaftsis, “Neutrinos and Collider Physics," New J. Phys. 17, no.7, 075019 (2015) doi:10.1088/13672630/17/7/075019 [arXiv:1502.06541 [hep-ph]].

[14] G. Cvetič, C. S. Kim and J. Zamora-Saá, “CP violations in $\pi^{ \pm}$Meson Decay,” J. Phys. G 41, 075004 (2014) doi:10.1088/0954-3899/41/7/075004 [arXiv:1311.7554 [hep-ph]].

[15] G. Cvetič, C. S. Kim and J. Zamora-Saá, “CP violation in lepton number violating semihadronic decays of $K, D, D_{s}, B, B_{c}$," Phys. Ref. D 89, no.9, 093012 (2014) doi:10.1103/PhysRevD.89.093012 [arXiv:1403.2555 [hep-ph]].

[16] C. O. Dib, M. Campos and C. S. Kim, "CP Violation with Majorana neutrinos in K Meson Decays," JHEP 02, 108 (2015) doi:10.1007/JHEP02(2015)108 [arXiv:1403.8009 [hep-ph]].

[17] G. Cvetič, C. Dib, C. S. Kim and J. Zamora-Saá, "Probing the Majorana neutrinos and their CP violation in decays of charged scalar mesons $\pi, K, D, D_{s}, B, B_{c}$," Symmetry 7, 726-773 (2015) doi:10.3390/sym7020726 [arXiv:1503.01358 [hep-ph]]

[18] G. Cvetič, C. S. Kim, R. Kogerler and J. Zamora-Saá, "Oscillation of heavy sterile neutrino in decay of $B \rightarrow \mu e \pi$, , Phys. Rev. D 92, 013015 (2015) doi:10.1103/PhysRevD.92.013015 [arXiv:1505.04749 [hep-ph]].

[19] G. Cvetič, C. S. Kim, S. Mendizabal and J. Zamora-Saá, "Exploring CP-violation, via heavy neutrino oscillations, in rare B meson decays at Belle II," Eur. Phys. J. C 80, no.11, 1052 (2020) doi:10.1140/epjc/s10052-020-08625-0 [arXiv:2007.04115 [hep-ph]].

[20] R. M. Godbole, S. P. Maharathy, S. Mandal, M. Mitra and N. Sinha, "Interference Effect in LNV and LNC Meson Decays for Left Right Symmetric Model," [arXiv:2008.05467 [hep-ph]]

[21] J. Zhang, T. Wang, G. Li, Y. Jiang and G. L. Wang, "The study of two quasi-degenerate heavy sterile neutrinos in rare meson decays," Phys. Rev. D 103, no.3, 035015 (2021) doi:10.1103/PhysRevD.103.035015 [arXiv:2010.13286 [hep-ph]].

[22] J. Zamora-Saá, “Resonant CP violation in rare $\tau^{ \pm}$decays," JHEP 05, 110 (2017) doi:10.1007/JHEP05(2017)110 [arXiv:1612.07656 [hep-ph]].

[23] S. Tapia and J. Zamora-Saá, “Exploring CP-Violating heavy neutrino oscillations in rare tau decays at Belle II," Nucl. Phys. B 952, 114936 (2020) doi:10.1016/j.nuclphysb.2020.114936 [arXiv:1906.09470 [hep-ph]].

[24] G. Aad et al. [ATLAS], "Measurement of $W^{ \pm}$and Z-boson production cross sections in $p p$ collisions at $\sqrt{s}=13$ TeV with the ATLAS detector," Phys. Lett. B 759, 601-621 (2016) doi:10.1016/j.physletb.2016.06.023 [arXiv:1603.09222 [hep-ex]].

[25] C. Degrande, O. Mattelaer, R. Ruiz and J. Turner, "Fully-Automated Precision Predictions for Heavy Neutrino Production Mechanisms at Hadron Colliders," Phys. Ref. D 94, no.5, 053002 (2016) doi:10.1103/PhysRevD.94.053002 [arXiv:1602.06957 [hep-ph]].

[26] A. Alloul, N. D. Christensen, C. Degrande, C. Duhr and B. Fuks, "FeynRules 2.0 - A complete toolbox for tree-level phenomenology," Comput. Phys. Commun. 185, 2250-2300 (2014) doi:10.1016/j.cpc.2014.04.012 [arXiv:1310.1921 [hep-ph]].

[27] J. Alwall, R. Frederix, S. Frixione, V. Hirschi, F. Maltoni, O. Mattelaer, H. S. Shao, T. Stelzer, P. Torrielli and M. Zaro, "The automated computation of tree-level and next-to-leading order differential cross sections, and their matching to parton shower simulations," JHEP 07, 079 (2014) doi:10.1007/JHEP07(2014)079 [arXiv:1405.0301 [hep-ph]].

[28] A. M. Sirunyan et al. [CMS], "Search for heavy neutral leptons in events with three charged leptons in proton-proton collisions at $\sqrt{s}=13$ TeV," Phys. Ref. Lett. 120, no.22, 221801 (2018) doi:10.1103/PhysRevLett.120.221801 [arXiv:1802.02965 [hep-ex]].

[29] V. Khachatryan et al. [CMS], "Search for heavy Majorana neutrinos in $\mu^{ \pm} \mu^{ \pm}+$jets events in proton-proton collisions at $\sqrt{s}=8 \mathrm{TeV}$," Phys. Lett. B 748, 144-166 (2015) doi:10.1016/j.physletb.2015.06.070 [arXiv:1501.05566 [hep-ex]].

[30] V. Khachatryan et al. [CMS], "Search for heavy Majorana neutrinos in $\mathrm{e}^{ \pm} \mathrm{e}^{ \pm}+$jets and $\mathrm{e}^{ \pm} \mu^{ \pm}+$jets events in proton-proton collisions at $\sqrt{s}=8$ TeV," JHEP 04, 169 (2016) doi:10.1007/JHEP04(2016)169 [arXiv:1603.02248 [hep-ex]]. 